African-American and European-Americans, and is located within an active enhancer region in neutrophils and modifies TREML4 expression. In vitro patch methylation experiments confirmed the regulatory effects of TREML4 methylation upon gene expression. Experiments to assess the functional effects of TREML4 overexpression in human neutrophils are underway in our laboratory. Interestingly, the DNA methylome was highly stable across disease activity levels and over time. Two sites cg26104306 (SNX18; FDR-adjusted P-value $=3.38 \mathrm{x}$ $10^{-2}$ ) and $\operatorname{cg} 06708913$ (FDR-adjusted P-value $=3.43 \times 10^{-2}$ ) were associated with changing disease activity levels in African-American patients. Demethylation of a $\mathrm{CpG}$ site located within GALNT18 was associated with the development of active lupus nephritis.

Conclusion Lupus granulocytes demonstrate significant differences in DNA methylation patterns between African-American and European-American patients. DNA methylation profiles in lupus patients are influenced by ancestry-specific genetic variants and are highly stable over time independent of disease activity levels. Progressive demethylation in SNX18 was observed with increasing disease activity in granulocytes from African-American lupus patients, and demethylation in GALNT18 was associated with the development of lupus nephritis in our cohort during follow up.

\section{SINGLE-CELL EPIGENETIC PROFILING HIGHLIGHTS GENETIC IMPACT ON CHROMATIN ACCESSIBILITY IN SLE}

1,2,3 3 ai Ma, ${ }^{4}$ Richard C Pelikan, ${ }^{4}$ Yao Fu, ${ }^{4}$ Jennifer A Kelly, ${ }^{4}$ David Murphy, ${ }^{4}$ Graham B Wiley, ${ }^{3}$ Vinay K Kartha, ${ }^{5}$ Caleb Lareau, ${ }^{2,3}$ Jason D Buenrostro, ${ }^{4}$ Patrick M Gaffney*. ${ }^{1}$ Department of Biology, MIT, Cambridge, MA, USA; ${ }^{2}$ Broad Institute of MIT and Harvard, Cambridge, MA, USA; ${ }^{3}$ Department of Stem Cell and Regenerative Biology, Harvard University, Cambridge, MA, USA; ${ }^{4}$ Genes and Human Disease Research Program, Oklahoma Medical Research Foundation, Oklahoma City, OK, USA; ${ }^{5}$ Department of Pathology, Stanford University, Stanford, CA, USA

\subsection{6/lupus-2021-lupus21century.91}

Background Chromatin accessibility (CA) is a critical epigenetic feature identifying genomic loci that actively participate in gene-regulating functions, such as transcription and DNA repair. In turn, genetic polymorphisms within these loci can affect the magnitude of CA, i.e. a chromatin accessibility quantitative trait locus (caQTL). Changes in CA have been implicated in inflammatory disease, and previous genetic research has identified several risk haplotypes for systemic lupus erythematosus (SLE). However, it remains unclear how CA may interact with genetic risk factors in SLE pathogenesis. To better understand how CA in SLE may be driven by genetic variation, we performed single-cell assay for transposase accessible chromatin (sciATAC-seq) on peripheral blood mononuclear cells (PBMCs). SciATAC-seq is an efficient, scalable and sensitive assay that allows the epigenetic profiling of thousands of cells from an individual. By combining these profiles with genotypic data, we can search for caQTLs as evidence of genetic-epigenetic interaction specific to SLE.

Methods PBMCs were isolated from 45 SLE patients and 50 healthy controls. Each PBMC sample underwent both genotyping and sci-ATAC sequencing. DNA was then sequenced by Illumina Next-seq. Cell-specific ATAC reads were demultiplexed and quantified by custom software developed by the BROAD Institute (Cambridge, MA). Genotyping data was phased and imputed using the IMPUTE2 tool suite. Cell typespecific caQTL analysis was performed by RASQUAL.

Results An average of 980 cells were sequenced per sample, with a total of 745,697 CA sites measured. We identified a total of 153,716 caQTL relationships across 17 distinct immune cell types, involving 59,715 unique variants; 59\% of which are also reported as expression QTLs in whole blood. The majority of caQTLs already implicated in autoimmune disease risk haplotypes occurred predominantly in B cells and plasmacytoid dendritic cells. CA profiles exhibit cell type-specific cluster orientation highly correlated with caQTL genotype. Genotypes at variant rs1131665, previously associated with an SLE risk haplotype in IRF7 and here as a caQTL, distinguished subpopulations of $\mathrm{B}$ cells and monocytes on the basis of global CA profiles. Increases of CA at the variant were present in SLE individuals compared to controls, suggesting caQTL variants contribute to a genome-wide epigenetic phenotype for SLE risk.

Conclusion Using advances in single-cell epigenetic profiling, we were able to identify thousands of genetic variants which influence epigenetic functions, in a cell type-specific way, through their association to CA. Understanding the molecular mechanisms for how caQTLs alter cell type-specific chromatin accessibility will provide new insights into the role of epigenetic regulation in SLE pathogenesis.

Acknowledgments The research reported in this abstract was supported by Institutional Development Awards (IDeA) from the National Institute of General Medical Sciences (U54GM104938 and P30GM110766), the National Institute of Arthritis and Musculoskeletal and Skin Diseases (P30AR073606 and R01AR073750); the National Institute of Allergy and Infectious Diseases (UM1AI144292 and R01AI156724), and by the Presbyterian Health Foundation (OKC, OK).

\section{DIFFERENCES IN CHROMATIN ARCHITECTURE PRE- AND POST-INDUCTION THERAPY IN PEDIATRIC LUPUS PATIENTS}

1,2,3 Joyce S Hui-Yuen ${ }^{*},{ }^{4,5}$ Kaiyu Jiang, ${ }^{3}$ Susan Malkiel, ${ }^{3}$ Betty Diamond, ${ }^{4,5}$ James N Jarvis. 'Division of Pediatric Rheumatology, Steven and Alexandra Cohen Children's Medical Center, Lake Success, NY, USA; ${ }^{2}$ Department of Pediatrics, Hofstra-Northwell School of Medicine, Hempstead, NY, USA; ${ }^{3}$ Center for Autoimmune, Musculoskeletal, and Hematologic Diseases Research, Feinstein Institute for Medical Research, Manhasset, NY, USA; ${ }^{4}$ Department of Pediatrics, University at Buffalo, Buffalo, NY, USA; ${ }^{5}$ Genetics, Genomics, and Bioinformatics Program, University at Buffalo, Buffalo, NY, USA

10.1136/lupus-2021-lupus21century.92

Background Systemic lupus erythematosus (SLE) may be triggered by gene-environment interactions. Data remain scarce on how epigenetic variance contributes to disease risk in pediatric SLE (pSLE). Our objectives were to identify differences in chromatin architecture in treatment-naïve pSLE compared to healthy children (HC) and pSLE patients after induction therapy.

Methods We used assays for transposase-accessible chromatinsequencing (ATACseq) in 8 pSLE patients pre- and post-induction therapy and $5 \mathrm{HC}$ to investigate whether regions of open chromatin unique to pSLE patients demonstrate enrichment for transcriptional regulators, using standard computational approaches and a false discovery rate of $<0.05$.

Results The mean age of onset was 13.75 (range 7-17) years in pSLE, and mean SLEDAI was 12.8 (range 6-24). We 
identified 245 differentially accessible regions (DAR) around peaks unique to treatment-naïve pSLE patients, of which over $50 \%$ appear to be more accessible in pSLE than HC, and are located more than $100 \mathrm{~kb}$ from the nearest transcription start site (nTSS), implying transcription factors (TF) may be acting on distal enhancers to regulate transcription. pSLE DAR were enriched for the enhancer $\mathrm{H} 3 \mathrm{~K} 4 \mathrm{me} 3$. In DAR encompassing TF binding sites, pSLE samples, but not HC, were enriched for several disease-associated SNPs previously identified in lupus genome-wide association studies. Variant calling within DAR found 3864 genes belonging to 129 different biologic processes, including cellular activation in immune response and responses to external stimuli. In contrast, over $80 \%$ of peaks unique to pSLE patients post-induction therapy are located distal to nTSS. Induction therapy for pSLE patients included corticosteroids in all patients, cyclophosphamide in 5, and mycophenolate in 3. DAR from the pSLE patients postinduction therapy were not enriched for enhancers or diseaseassociated SNPs.

Conclusion We demonstrate an epigenetically-distinct profile in pSLE B cells when compared to HC, indicating pSLE B cells are predisposed for disease development. Pathways of significance analyses identified immunologic pathways important in the pro-inflammatory response in treatment-naïve pSLE patients. These pathways were absent in analyses from the same pSLE patients post-induction therapy. Thus, increased chromatin accessibility in genomic regions controlling activation of inflammatory and immune responses suggest transcriptional dysregulation of key players in immune cell activation plays an important role in pathogenesis of SLE. Treatment with corticosteroids and immunosuppressive medication changes this epigenetic profile, making pathways responsible for inflammation and B cell activation less accessible.

Acknowledgment This work would not have been possible without the expertise of Dr Frank Jenkins. This work was funded by the Rheumatology Research Foundation.

\section{PREDICTING RISK OF SEVERE LUPUS NEPHRITIS IN AFRICAN AMERICANS: THE APOL1 STORY}

Barry I Freedman*. Section on Nephrology, Wake Forest School of Medicine; WinstonSalem, NC, USA

\subsection{6/lupus-2021-lupus21century.93}

Background Compared to European, Asian and Native Americans, African Americans have a 3-fold higher risk of developing end-stage kidney disease (ESKD) and are more likely to develop severe lupus nephritis (LN).

Methods Strong genetic association is observed between the apolipoprotein L1 gene (APOL1) and a spectrum of non-diabetic chronic kidney diseases (CKD) in African Americans, including LN, focal segmental glomerulosclerosis, solidified glomerulosclerosis (hypertension-attributed nephropathy), HIVassociated nephropathy, sickle cell nephropathy, and premature failure of transplanted kidneys from APOL1 high-risk donors. APOL1 risk variants arose in sub-Saharan Africa and are present only in those who possess recent African ancestry. These variants account for much of the excess risk for LN and $\mathrm{CKD}$ in African Americans. Studies in transgenic mice prove that APOL1 risk variants cause CKD. Kidney disease is due to locally produced APOL1 protein in kidney cells, not circulating APOL1 protein in the blood.
Results Patients with systemic lupus erythematosus who inherit two APOL1 risk variants are more likely to progress to ESKD and often display focal and diffuse proliferative or membranous glomerular lesions. Kidney disease often progresses despite cytotoxic therapy. In contrast, APOL1 is not associated with mild LN. Therefore, APOL1 risk variants are nephropathy progression factors. Not all individuals with two APOL1 risk variants develop $\mathrm{CKD}$; modifying factors are required. HIV infection, SARS-CoV-2 infection, and interferon are powerful second hits that initiate nephropathy in genetically susceptible hosts.

Conclusions Conventional treatments for kidney disease often fail to halt the progression of non-diabetic CKD. Novel small molecule inhibitors of APOL1 protein and APOL1 anti-sense oligonucleotides hold great promise for slowing progression of APOL1-associated nephropathy, including LN. Treatments have the potential to reduce disparities in CKD risk among individuals with African ancestry. In addition, the NIH 'APOL1 Long-term Kidney Transplant Outcomes' (APOLLO) Consortium is considering the role of APOL1 genotyping in deceased African American kidney donors to improve organ allocation. Discovery of the APOL1 genetic association with nephropathy in the lab has moved to the bedside and will improve patient outcomes.

\section{0 - Biomarkers in clinical trials}

\section{SCORING PERSONALIZED MOLECULAR PORTRAITS OF SYSTEMIC LUPUS ERYTHEMATOSUS PATIENTS TO PREDICT TREATMENT RESPONSES, FLARES, AND PROGNOSIS}

1Daniel Toro-Domínguez, 1,2Raúl López-Domínguez, ${ }^{1}$ Guillermo Barturen, ${ }^{1,2}$ Pedro Carmona-Sáez ${ }^{\dagger},{ }^{1,3}$ Marta E Alarcón-Riquelme* ${ }^{*} .{ }^{1}$ GENYO, Granada, Spain; ${ }^{2}$ University of Granada, Granada, Spain; ${ }^{3}$ Karolinska Institute, Stockholm, Sweden

10.1136/lupus-2021-lupus21 century.94

Objectives Systemic Lupus Erythematosus is a complex autoimmune disease that leads to important worsening of the quality of life and significant suffering to those affected. Currently, therapies used are partially inefficient, mainly due to the molecular heterogeneity of the disease, being personalized medicine the big promise for the future of autoimmunity. With this work we intend to take a step further in that direction by developing MyPROSLE, a system capable of measuring the molecular portrait of individual patients.

Methods We defined co-expressed and functionally annotated gene-modules conserved across two longitudinal datasets with 158 and 301 patients. The dysregulation magnitude of each gene-module was calculated at the patient level using averaged z-scores. We analyzed the association between gene-modules, clinical manifestations and the evolution of the disease by ANOVA, Student's t-test and Cox proportional-hazard models. Drug responses to hydroxychloroquine and mycophenolate was analyzed by comparing each individual's molecular portraits. A third dataset of 1760 patients was used to compare the response to Tabalumab.

Results The system allows to quantify the dysregulation of 30 gene-modules individually with respect to healthy distributions. We show that dysregulation of certain gene-modules is strongly associated with different clinical manifestations and with predicting the time when remissions and relapses of the 